\title{
Charge ordering via electron-electron interactions in the colossal-magnetoresistive manganites
}

\author{
S. K. Mishra, Rahul Pandit, ${ }^{*}$ and Sashi Satpathy \\ Department of Physics and Astronomy, University of Missouri, Columbia, Missouri 65211
}

(Received 12 February 1997)

\begin{abstract}
The coupled magnetic and charge-order transition observed in the manganites of the type $R_{1-x} M_{x} \mathrm{MnO}_{3}$ near half filling $(x \simeq 1 / 2)$ is shown to be the result of the interplay between the doubleexchange, superexchange, and the Coulomb interaction terms in an electronic Hamiltonian. At half filling and temperature $T=0$ we find, as we increase the strength of the extended-Hubbard repulsion, a first-order transition from a charge-nonordered ferromagnetic metal $(\mathrm{FN})$ to a charge-ordered antiferromagnetic and insulating (AFO) ground state. The AFO-FN transition is also obtained by increasing $T$; however, a small degree of charge order remains in the ferromagnetic phase. The charge-ordered state also "melts," as observed, on the application of a magnetic field, which causes a rapid drop in the transition temperature. Qualitative differences in behavior between members of the manganite series can be understood in terms of small variations in the interaction parameters. [S0163-1829(97)00930-2]
\end{abstract}

The observation ${ }^{1}$ of colossal magnetoresistance (CMR) in manganites, which provide the classic examples of the double-exchange magnetic coupling, ${ }^{2-4}$ has generated new interest in their intriguing properties. These are compounds of the form $R_{1-x} M_{x} \mathrm{MnO}_{3} \quad(R=\mathrm{La}, \mathrm{Nd}$, or $\operatorname{Pr}$ and $M=\mathrm{Sr}$, $\mathrm{Ca}, \mathrm{Ba}$, or $\mathrm{Pb}$ ), which were studied extensively in the $1950 \mathrm{~s}^{5-7}$ In addition to the CMR, other noteworthy properties include (1) a close competition between ferromagnetic phases, favored by the double-exchange mechanism, antiferromagnetic phases, favored by superexchange and correlation effects, and a paramagnetic phase, which appears at high temperatures $T{ }^{7-13}$ (2) at half filling, $x=0.5$, this charge ordering can be thought of nominally as $\mathrm{Mn}^{3+}$ ions on one sublattice and $\mathrm{Mn}^{4+}$ ions on the other, superimposed on any magnetic ordering that might exist. For instance, $\mathrm{Nd}_{0.5} \mathrm{Sr}_{0.5} \mathrm{MnO}_{3}$ and $\mathrm{La}_{0.5} \mathrm{Ca}_{0.5} \mathrm{MnO}_{3}$ are charge-ordered antiferromagnets 7,11 (AFO's) with the "CE" crystal structure (Fig. 1) at low $T$; on increasing $T$ they go to a ferromagnetic phase via a first-order transition. The AFO phase is insulating but the ferromagnetic phase is metallic. On further increasing $T$, the ferromagnetic phase goes to an insulating paramagnetic phase via a continuous transition. (3) The AFO-FN transition temperature $T_{\mathrm{AF} \rightarrow \mathrm{F}}$ falls rapidly with increasing magnetic field $H$, and (4) at fixed $T$, the AFO phase can be "melted" by the application of the magnetic field.

Theoretical studies of these manganites have concentrated on the double-exchange mechanism, ${ }^{2-4}$ the effects of electron-phonon interactions, ${ }^{14}$ spin-polaron and offdiagonal localization effects, ${ }^{15}$ and on spiral, ${ }^{16}$ canted, ${ }^{4,17}$ or spin and orbital ${ }^{18}$ orderings. The relative importance of the various interactions on the physical properties of the manganites is currently the subject of a lively debate.

In this paper, we show that the coupled magnetic and charge-order transition observed in these materials, at or near half filling, is described well by an electronic Hamiltonian containing the Hubbard and the extended-Hubbard interactions in addition to the double-exchange term. Our model does not contain any explicit electron-phonon coupling, although the static Jahn-Teller distortion is used to argue for the simple one-band model we use.
The salient features of the electronic structures ${ }^{19,20}$ of the manganites can be summarized as follows: The important bands near the Fermi energy $E_{f}$ consist of $\mathrm{Mn}_{3 d}$ states. In $\mathrm{LaMnO}_{3}$ the $\mathrm{Mn}$ atom is trivalent $\left[t_{2 g}(3) e_{g}(1)\right.$ majorityspin configuration], with the half-filling of the doubly degenerate $e_{g}$ band leading to a Jahn-Teller distortion, which, in turn, splits off the $e_{g}$ band into a lower $e_{g}(1)$ band and an upper $e_{g}(2)$ band leading to an insulator within the band theory. In $\mathrm{CaMnO}_{3}$ the $\mathrm{Mn}$ atom is tetravalent, with the $t_{2 g}(3)$ majority-spin configuration. The $\mathrm{Ca}$ dopant in $\mathrm{La}_{1-x} \mathrm{Ca}_{x} \mathrm{MnO}_{3}$ therefore introduces holes into the $e_{g}(1)$ band. This band being about $2 \mathrm{eV}$ wide, the doped electrons (holes) may be considered as itinerant, which interact via a Hund's-rule coupling with the localized $t_{2 g}$ electrons $S=\frac{3}{2}$, leading to a double-exchange coupling that tends to align the $t_{2 g}$ spins ferromagnetically. It is this competition between

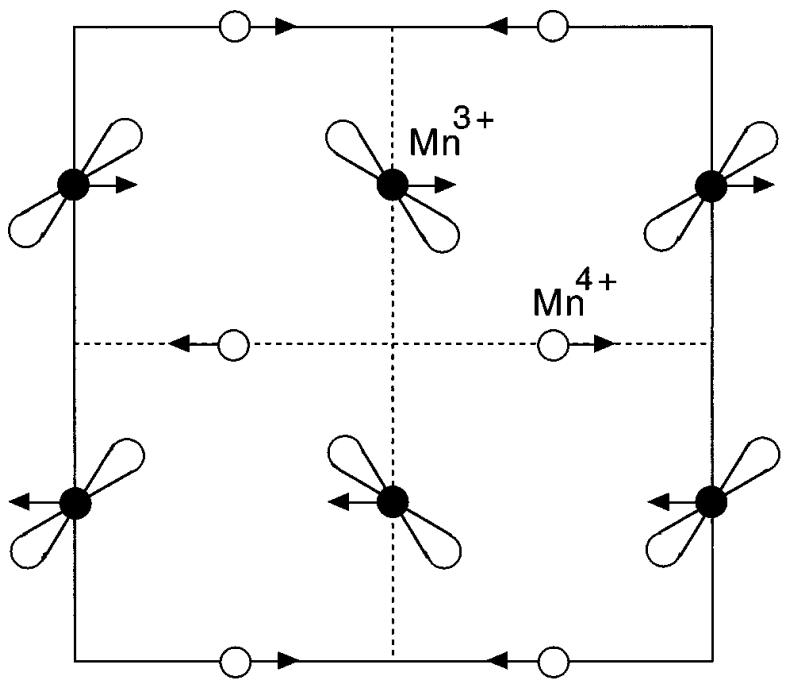

FIG. 1. The spin and charge configurations on the Mn sites in the antiferromagnetic charge-ordered $\mathrm{CE}$ structure on the $a b$ plane. Such planes are stacked along the $c$ direction with the spins reversed on the successive planes. Sites with charge deficits and excesses are indicated, respectively, by $\bigcirc$ and 0 . 
the double-exchange term and the ordinary superexchange between the localized spins, in addition to the Coulomb interaction, that is responsible for the rich variety of phenomena exhibited by these manganites, as we shall show. It is our point of view that the charge ordering is determined principally by the interacting electron system, with the electronphonon coupling, apart from the static Jahn-Teller distortion, merely serving to renormalize the electronic parameters.

We consider the following Hamiltonian which describes the system of localized $t_{2 g}$ spins and the itinerant electrons in the Jahn-Teller split $e_{g}(1)$ band:

$$
\begin{aligned}
\mathcal{H}= & -t \sum_{\langle i j\rangle, \sigma} c_{i \sigma}^{\dagger} c_{j \sigma}+\text { c.c. }+U_{0} \sum_{i} \hat{n}_{i \uparrow} \hat{n}_{i \downarrow}+U_{1} \sum_{\langle i j\rangle, \sigma \nu} \hat{n}_{i \sigma} \hat{n}_{j \nu} \\
& -J_{\mathrm{ex}} \sum_{\langle i j\rangle} \mathbf{S}_{i} \cdot \mathbf{S}_{j}-2 J_{H} \sum_{i} \mathbf{S}_{i} \cdot \mathbf{S}_{i}-\mu \sum_{i \sigma} \hat{n}_{i \sigma} \\
& -g \mu_{B} \mathbf{H} \cdot \sum_{i}\left(\mathbf{S}_{i}+\mathbf{s}_{i}\right) .
\end{aligned}
$$

Thus we have, in addition to a lattice version of the Anderson-Hasegawa Hamiltonian, a magnetic field and Coulomb-interaction terms between the itinerant electrons. The chemical potential $\mu$ is used to control the number of electrons, $t$ is the hopping amplitude between nearestneighbor sites $\langle i j\rangle, c_{i \sigma}^{\dagger}$ and $c_{i \sigma}$ are, respectively, creation and annihilation operators for electrons on site $i$ with spin $\sigma, \hat{n}_{i \sigma}=c_{i \sigma}^{\dagger} c_{i \sigma}, \mathbf{H}$ is the external magnetic field, $\mathbf{S}_{i}$ is the localized Mn spin, $\mathbf{s}_{i} \equiv \frac{1}{2} \Sigma_{\sigma \nu}\left(c_{i \sigma}^{\dagger} \tau_{\sigma \nu} c_{i \nu}\right)$ is the conductionelectron spin density with $\tau$ being the Pauli matrices, $J_{H}$ is the Hund-rule coupling, $J_{\mathrm{ex}}<0$ is the antiferromagnetic superexchange coupling between the localized spins, and $U_{0}$ and $U_{1}$ are, respectively, the on-site and the nearest-neighbor Hubbard repulsion terms. We set $S=\frac{3}{2}$. From densityfunctional studies we estimate ${ }^{19} t \simeq 0.15 \mathrm{eV}, J_{H} S \simeq 0.75 \mathrm{eV}$, $U_{0} \simeq 10 \mathrm{eV}$, and $J S^{2} \simeq 8 \mathrm{meV} .{ }^{21}$ We estimate $U_{1} \simeq e^{2} /$ $(\epsilon r) \simeq 0.3-0.4 \mathrm{eV}$ for these manganites by assuming $\epsilon \simeq 10$ and $r \simeq 4 \AA$. It is this term which favors chargeordered phases as in the Cullen-Callen model ${ }^{22}$ for the Verwey transition in magnetite; however, the charge-ordering phenomenon in the manganites is more complex due to the coupling between the charge and the spin degrees of freedom.

We study the Hamiltonian (1) in the Hartree approximation, which is set up most conveniently by using the GibbsBogoliubov-Peierls variational principle ${ }^{25}$ for both $T=0$ and $T>0$. This yields self-consistency equations for the order parameters, which we solve numerically. We select the solution which gives the lowest minimum of variational grand free energy $\Omega_{\mathrm{var}}$. At $T=0$, this is equivalent to minimizing the variational energy $E_{\mathrm{var}}$ for a fixed filling $x$. The order parameters $M_{i} \equiv\left\langle\mathbf{S}_{i}\right\rangle \quad$ (scaled by $S$ ), satisfy the selfconsistency equations

$$
r_{i}=B_{S}\left[\beta\left(J_{\mathrm{ex}} S^{2} \sum_{j \prime}{ }^{\prime} M_{j}+J_{H} S m_{i}+g \mu_{B} H S\right)\right],
$$

where $\beta \equiv\left(k_{B} T\right)^{-1}, k_{B}$ is the Boltzmann constant, the prime on the sum denotes nearest neighbors of site $i$, the spin density $m_{i} \equiv\left\langle\hat{n}_{i \uparrow}-\hat{n}_{i \downarrow}\right\rangle$, and $B_{S}$ is the Brillouin function. The

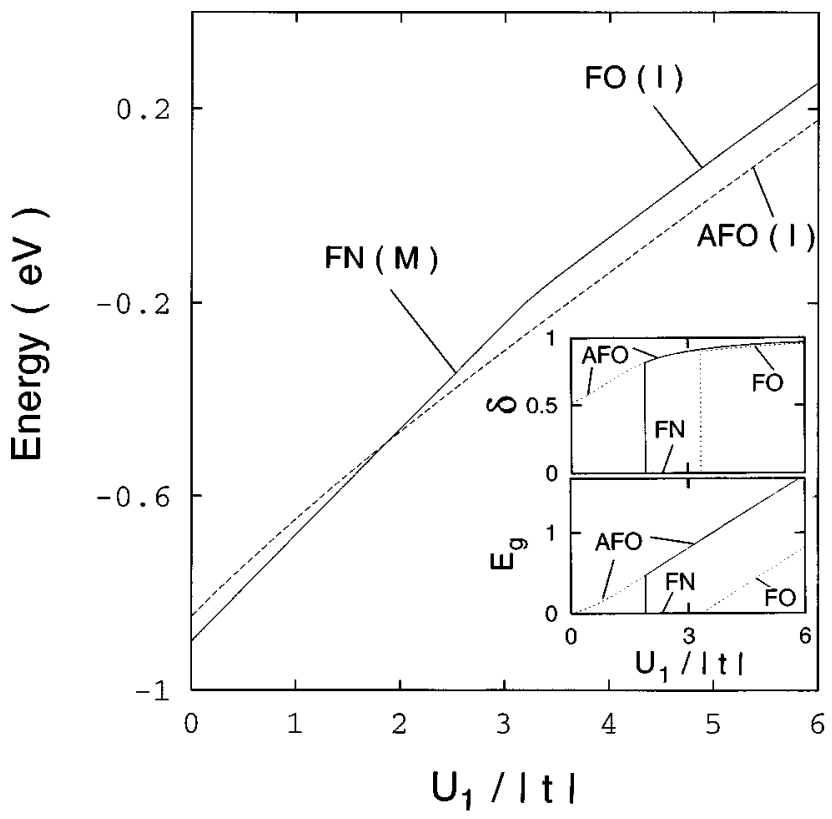

FIG. 2. Variation of the energies of the ferromagnetic and antiferromagnetic structures at $T=0$ with the nearest-neighbor Coulomb repulsion $U_{1}$ at half filling. Parameters used here are $J_{H}=0.4 \mathrm{eV}, J_{\mathrm{ex}}=10 \mathrm{meV}, U_{0}=10 \mathrm{eV}$, and $t=0.15 \mathrm{eV}$. The double-exchange mechanism always favors the ferromagnetic state. At larger $U_{1}$ the AF state wins because the superexchange term ultimately dominates as the kinetic energy gain by the doubleexchange mechanism is weakened with increasing $U_{1}$. Insets show the variation of the charge-order parameter $\delta$ and the one-particle gap $E_{g}$ with $U_{1}$. Dotted lines in the inset show the same quantities for the individual ferromagnetic and the AF phases.

charge densities $n_{i \sigma} \equiv\left\langle\hat{n}_{i \sigma}\right\rangle$ are determined self-consistently from the eigenvectors of the effective mean-field Hamiltonian. For the CE structure of Fig. 1 this requires the diagonalization of a $32 \times 32$ matrix for each point in the Brillouin zone. If $H=0$, there are a total of six order parameters in our model, viz., $M_{i}, m_{i}$, and $n_{i}=n_{i \uparrow}+n_{i \downarrow}$, with $i=\bigcirc$ or $i=0$, the two types of sites in Fig. 1 (for $H>0,12$ order parameters are required).

At $T=0$ four phases compete: ferromagnetic chargeordered (FO) or charge-nonordered (FN), and antiferromagnetic charge ordered (AFO) or charge-non-ordered (AFN) phases. We show in Fig. 2 how the mean-field energies for these phases vary with $U_{1}$ at half filling. The ground state is determined by the phase with the lowest energy, so that as seen from the figure at $U_{1} / t \simeq 1.9$, there is a first-order FNAFO transition. This illustrates clearly how the extendedHubbard repulsion $U_{1}$ promotes charge ordering, which since the charge and the spin degrees of freedom are coupled, also leads to a magnetic transition. Note that our estimate of the magnitude of $U_{1}$ is close to the FN-AFO transition point in Fig. 2, leading to a natural explanation for why some of these systems (e.g., $\mathrm{La}_{0.5} \mathrm{Sr}_{0.5} \mathrm{MnO}_{3}$ ) display a FN phase at $x=0.5$, whereas others (e.g., $\mathrm{La}_{0.5} \mathrm{Ca}_{0.5} \mathrm{MnO}_{3}$ ) are in the AFO phase. The top and bottom insets in Fig. 2 display, respectively, how the charge-order parameter $\delta \equiv\left(n_{\bullet}-n_{\bigcirc}\right)$ and the gap $E_{g}$ jump from zero, in the FN phase, to finite values in the AFO phase. At half filling, 


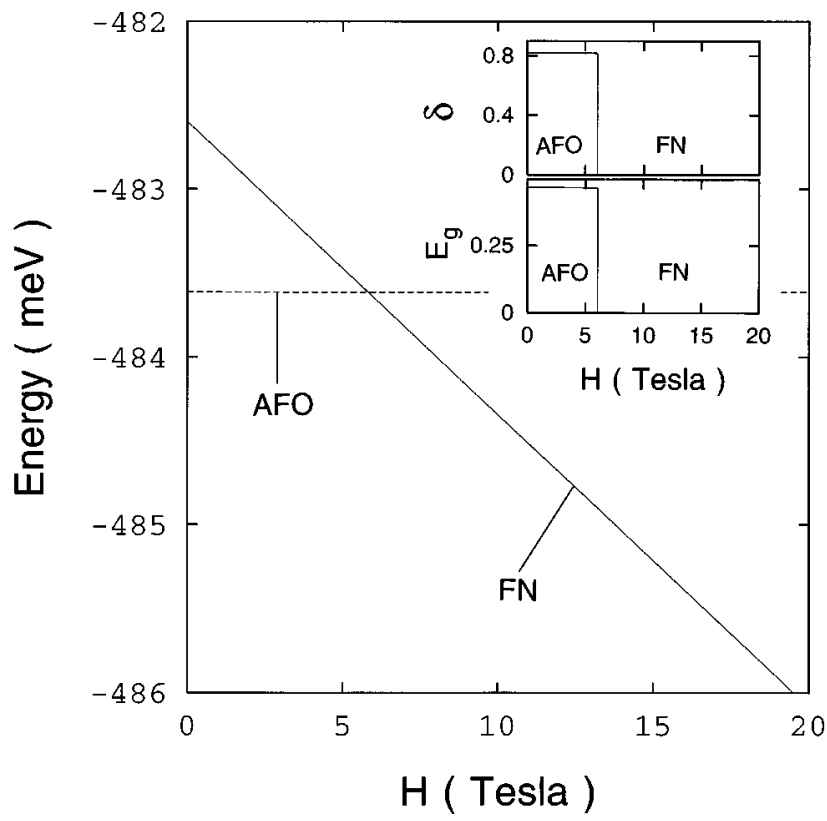

FIG. 3. Variation of the energies of the AF and the ferromagnetic phases indicating the "melting" of the charge-ordered state into a charge-nonordered state induced by an applied magnetic field $H$. This behavior is consistent with the experimental observations in systems such as $\mathrm{Nd}_{0.5} \mathrm{Sr}_{0.5} \mathrm{MnO}_{3}$ and $\mathrm{Pr}_{0.5} \mathrm{Sr}_{0.5} \mathrm{MnO}_{3}$. The variation of the charge-order parameter and the gap with $H$ are shown in the inset. Here, $U_{1}=0.285 \mathrm{eV}$ and other parameters are the same as in Fig. 2, resulting in an AFO state at $T=0$.

therefore, we have either a metallic FN or an insulating AFO phase, separated by a first-order boundary. We can also cross the AFO-FN transition by applying a weak magnetic field $H$, as illustrated in Fig. 3. This field-induced "melting" of the charge order is in accord with experiments. 9,11

The $T=0$ AFO-FN transition is replaced at $T>0$ by a first-order AFO-FO transition at $x=1 / 2$ (Fig. 4). Even

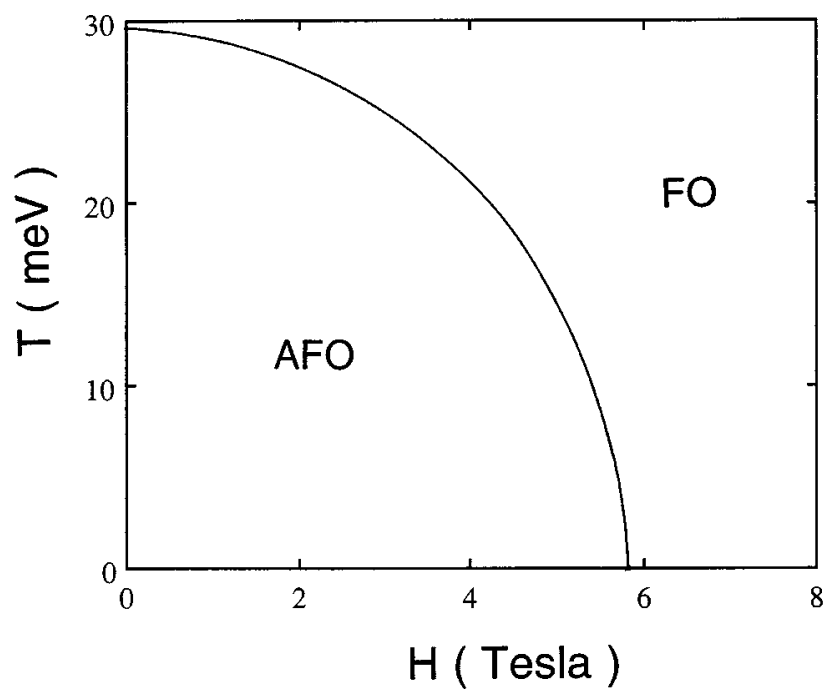

FIG. 4. Calculated magnetic phase boundary, for the parameters of Fig. 3, indicating transition between the AFO and the FO states. There is a large jump in the charge-order parameter across the phase boundary with the FO phase having only a small charge order, $\delta \approx 0.2$, as discussed in the text. though we find both phases to be charge ordered, there is a large jump in the charge-order parameter $\delta$ in going from the AFO to the FO phase. For physically reasonable parameters, the degree of charge ordering in the FO phase is quite small: In Fig. 4, this jump amounts to $\delta \approx 0.9$ in the AFO phase to about 0.2 in the FO phase. The ferromagnetic moment $M \equiv\left(M_{\bullet}-M_{\odot}\right)$ is nearly saturated at the transition point. We find that both $\delta$ and $M$ rise slightly before the transition. It would be interesting to check whether such effects are related to the possible presence of multiple intermediate phases reported recently. ${ }^{13}$

The AFO phase in our model exists in a narrow range near $x=1 / 2$ and is bounded on both sides by two-phase $(\mathrm{AFO}+\mathrm{FO})$ coexistence regimes, which meet at a point of equal concentration at $T=T_{\mathrm{AF} \rightarrow \mathrm{F}}(\simeq 0.03 \mathrm{eV}$ for the parameters of Fig. 4). We shall present details of such phase diagrams elsewhere ${ }^{24}$ On further increasing $T$, the degree of charge ordering in the FO phase increases, until a first-order FO-FN transition occurs at $T \simeq 0.2 \mathrm{eV}$, and, at much higher temperatures, a continuous FN-PN transition; we believe that the fluctuations negelected by our mean-field theory should lower the FN-PN transition temperature substantially. Note also that experiments are done at a fixed filling; if at some $T$ the filling $x$ lies in a region of two-phase coexistence, phase separation should occur and the equilibrium state should be comprised of two phases, separated by an interface, and with fillings specified by the boundaries of the coexistence curve at that value of $T$. Such an equilibrium state might be hard to obtain in experiments, since dopantatom diffusion might be kinetically hindered; furthermore, data analysis may be complicated by the possible presence of multiple intermediate phases. ${ }^{13}$

To obtain correct structures, changes in lattice parameters at these transitions, or charge-ordered phases interpreted as polaronic crystals, ${ }^{26}$ one must of course include other interactions. By virtue of using a single-band model, motivated by the Jahn-Teller splitting of the $e_{g}$ band, we have implicitly accounted for some degree of electron-phonon interactions; clearly a more detailed modeling of these interactions is required to obtain charge-ordered phases that can be interpreted as polaronic crystals. We note that, once charge ordering sets in because of the repulsion term $U_{1}$, it will induce a Jahn-Teller-type frozen-phonon distortion, i.e., a polaronic crystal. Also further-neighbor superexchange terms, that should destabilize other phases like a conventional twosublattice antiferromagnet, might well have to be included to make the $\mathrm{CE}$ structure the most stable one near $x=1 / 2$. But then one should use a multiband model and perhaps also different values for in-plane and out-of-plane hopping amplitudes, in view of the in-plane orientation of the $e_{g}^{(1)}$ orbital and the orbital ordering. ${ }^{18,19,23}$ In our study we have only considered the CE structure (Fig. 1) for the AFO phase which is the experimentally observed structure at half filling.

Our paramagnetic phase is metallic, though in experiments it is insulating (even away from commensurate fillings). Clearly disorder effects (both spin disorder ${ }^{15}$ and disorder arising from doping) must be included to make the $\mathrm{AFO}$ and PN phases insulating at away from half filling; electron-phonon interactions should also be important. ${ }^{14} \mathrm{We}$ also note that the $H$-driven AFO-FN insulator-to-metal tran- 
sition would obviously be associated with a colossal magnetoresistance, but this is different from the conventional CMR associated with the FN-paramagnetic insulator transition. Our results indicate that, in addition to the double-exchange and electron-phonon interactions, ${ }^{14}$ one would have to include the electron-electron interactions that stabilize the AFO phase, since it responds so sensitively to a magnetic field. In summary, then, our study elucidates the competition between charge-ordered and other magnetically ordered phases in the CMR manganites, bringing out the crucial role of electron-electron interactions in stabilizing the charge order.

This work was supported in part by the Office of Naval Research under Contract No. ONR N00014-95-1-0439. We thank H. R. Krishnamurthy, R. Mahendiran, and T. V. Ramakrishnan for discussions.
*Permanent address: Department of Physics, Indian Institute of Science, Bangalore - 560 012, India. Also at Jawaharlal Nehru Center for Advanced Scientific Research, Bangalore, India.

${ }^{1}$ R. von Helmolt, J. Weckberg, B. Holzapfel, L. Schultz, and K. Samwer, Phys. Rev. Lett. 71, 2331 (1993); K. Chahara, T. Ohno, M. Kasai, and Y. Kozono, Appl. Phys. Lett. 63, 1990 (1993); S. Jin, T. H. Tiefel, R. A. Fastnacht, R. Ramesh, and L. H. Chen, Science 264, 413 (1994).

${ }^{2}$ C. Zener, Phys. Rev. 82, 403 (1951).

${ }^{3}$ P. W. Anderson and H. Hasegawa, Phys. Rev. 100, 675 (1955).

${ }^{4}$ P.-G. de Gennes, Phys. Rev. 118, 141 (1960).

${ }^{5}$ G. H. Jonker and J. H. Van Santen, Physica (Amsterdam) 16, 337 (1950); 16, 599 (1950).

${ }^{6}$ E. O. Wollan and W. C. Koehler, Phys. Rev. 100, 545 (1955).

${ }^{7}$ J. B. Goodenough, Phys. Rev. 100, 564 (1955).

${ }^{8}$ I. O. Troyanchuk, Zh. Eksp. Teor. Fiz. 102, 251 (1992) [Sov. Phys. JETP 75, 132 (1992)].

${ }^{9}$ Y. Tomioka, A. Asamitsu, Y. Moritomo, H. Kuwahara, and Y. Tokura, Phys. Rev. Lett. 74, 5108 (1995).

${ }^{10}$ K. Knizek, Z. Jirak, E. Pollert, F. Zounova, and S. Vratislav, J. Solid State Chem. 100, 292 (1992); Y. Tomioka, A. Asamitsu, H. Kuwahara, Y. Moritomo, and Y. Tokura, Phys. Rev. B 53, R1689 (1996).

${ }^{11}$ H. Kuwahara, Y. Tomioka, A. Asamitsu, Y. Moritomo, and Y. Tokura, Science 270, 961 (1995); T. Vogt, A. K. Cheetham, R. Mahendiran, A. K. Raychaudhuri, R. Mahesh, and C. N. R. Rao, Phys. Rev. B 54, 15303 (1996).

${ }^{12}$ K. Liu, X. W. Wu, K. H. Ahn, T. Sulchek, C. L. Chien, and John
Q. Xiao, Phys. Rev. B 54, 3007 (1996).

${ }^{13}$ P. G. Radaelli, D. E. Cox, M. Marezio, S-W. Cheong, P. E. Schiffer, and A. P. Ramirez, Phys. Rev. Lett. 75, 4488 (1995).

${ }^{14}$ A. J. Millis, P. B. Littlewood, and B. I. Shraiman, Phys. Rev. Lett. 74, 5144 (1995); A. J. Millis, B. I. Shraiman, and R. Mueller, ibid. 77, 175 (1996).

${ }^{15}$ C. M. Varma, Phys. Rev. B 54, 7328 (1996).

${ }^{16}$ J. Inoue and S. Maekawa, Phys. Rev. Lett. 74, 3407 (1995).

${ }^{17}$ S. K. Mishra, S. Satpathy, F. Aryasetiawan, and O. Gunnarson, Phys. Rev. B 55, 2725 (1997).

${ }^{18}$ S. Isihara, J. Inoue, and S. Maekawa (unpublished); W. Koshibae, Y. Kawamura, S. Isihara, S. Okamuto, J. Inoue, and S. Maekawa (unpublished).

${ }^{19}$ S. Satpathy, Z. S. Popović, and F. R. Vukajlović, Phys. Rev. Lett. 76, 960 (1996).

${ }^{20}$ W. E. Pickett and D. J. Singh, Phys. Rev. B 53, 1146 (1996); D. D. Sarma, N. Shanthi, S. R. Barman, N. Hamada, H. Sawada, and K. Terakura, Phys. Rev. Lett. 75, 1126 (1995).

${ }^{21}$ I. Solovyev (private communication).

${ }^{22}$ J. R. Cullen and E. R. Callen, Phys. Rev. B 7, 397 (1973).

${ }^{23}$ V. I. Anisimov, I. S. Elfimov, M. A. Korotin, and K. Terakura (unpublished).

${ }^{24}$ S. K. Mishra, R. Pandit, and S. Satpathy (unpublished).

${ }^{25}$ M. Girardeau and R. Mazo, in Advances in Chemical Physics, edited by I. Prigogine and S. A. Rice (Wiley, New York, 1973), pp. 187-255.

${ }^{26}$ Y. Yamada, O. Hino, S. Nohdo, R. Kanao, T. Inami, and S. Katano, Phys. Rev. Lett. 77, 904 (1996). 